\title{
Factors Influencing the Onset of Neoadjuvant Therapy in Breast Cancer Patients
}

\author{
Carolin Müller Stephanie Juhasz-Böss Gilda Schmidt Erich-Franz Solomayer \\ Ingolf Juhasz-Böss Georg-Peter Breitbach \\ Department of Gynecology, Obstetrics and Reproductive Medicine, Saarland University Medical School, Homburg, \\ Germany
}

\section{Keywords}

Breast cancer - Neoadjuvant therapy - Therapy onset . Delaying factors $\cdot$ Study participation

\begin{abstract}
Background: Time to treatment onset (TTTO) is critical in breast cancer patients receiving neoadjuvant chemotherapy (NACT). We therefore investigated possible delaying factors of therapy onset. Methods: All patients were included who qualified for NACT in our hospital from 2015 to 2017. The time interval between core biopsy of tumor and date of therapy onset was defined as primary endpoint. Among other things, age, out- and in-patient presentation, and study or standard treatment were investigated as potentially delaying factors. Results: We analyzed 139 patients scheduled for NACT; 90 (64.7\%) received standard NACT, and 49 (35.3\%) were recruited for trials. The average age was 53 years $( \pm 13.2$ years). A time interval of 30.7 days ( \pm 11.8 days) was seen between diagnosis and therapy onset. Patients had a mean of $5( \pm 1.9)$ pretherapeutic presentations, $4( \pm 1.8)$ on outpatient and $1( \pm 0.5)$ on inpatient basis, being of significant influence on TTTO. Conclusion: Any outpatient presentation extended the time interval by 2 days, inpatient presentation by 4 days. These presentations should be merged in order to minimize TTOO. Neither the site of pathology examinations, additional consultations (genetics, reproductive medicine), nor study participation delayed therapy onset.
\end{abstract}

(C) 2019 S. Karger AG, Basel

\section{Background}

Breast cancer (BC) is the most frequent malignant disease in women, and in Germany the lifetime risk adds up to $1: 8$. A total of 71,640 new cases were reported 2013, and only $1 \%$ of them were attributed to male patients. The incidence of BC has increased over the last years so that in 2020 about 77,600 new diseases may be expected. Thanks to many new successful therapy strategies, BC mortality has dropped since 1990. Currently, 5-year survival is estimated at $88 \%$ and 10 -year survival at $82 \%$ [1]. Neoadjuvant therapy (NACT) has been developed as an important therapeutic option. If chemotherapy seems mandatory due to diagnostic and prognostic parameters, it should be scheduled in a neoadjuvant setting $[2,3]$. Historically, NACT has been used in inflammatory, primarily inoperable and locally advanced BC $[2,4]$. According to guidelines, NACT should include anthracyclines and taxanes [2]. Modified therapy schedules should only be used in a clinical study setting. Regardless of the planned schedule, an early onset of therapy is desirable. We therefore investigated several possible delaying factors of therapy onset.

\section{Patients and Methods}

Data Collection

Data of all patients that were scheduled for NACT in the period from 2015 to 2017 were collected. All therapy schedules were planned by the tumor board in the certified Breast Cancer Center of the Department of Gynecology, Saarland University Medical School. Tu- 
mor board decision followed complete diagnosis and determination of prognostic factors. The primary endpoint was the time interval in between the day of core biopsy of the breast tumor and the first day of systemic therapy application (time to treatment onset, TTTO).

Patients' age, stage, eventual multicentricity and tumor biology of $\mathrm{BC}$ were recorded. Another factor of possible influence on time interval was supposed to be participation in a clinical study or standard therapy. From 2015 to 2017, the following German neoadjuvant study designs were addressed: GAIN II, GeparOcto, GeparX, Nadens, and Adapt studies. The number of presentations in the hospital was recorded, either on outpatient or inpatient basis. It was recorded whether pathology examination was performed at external or internal institutions. Data of genetic counseling and reproductive advice were collected as well as fertility preservation procedures. Primary BC was separated from secondary contralateral $\mathrm{BC}$, and double carcinoma combined with a second primary tumor was documented.

\section{Data Management}

Patients' data were reviewed in the hospital's digital documentation system (SAP, Walldorf, Germany). Data were collected using Microsoft Excel 2010 ${ }^{\circledR}$ (Microsoft, Redmond, WA, USA). Further statistics were performed with SPSS 24.0 (IBM, Armonk, NY, USA) was used. Quantitative parameters like time intervals, patients' age, and number of clinical presentations are presented as mean \pm SD. Qualitative parameters like tumor biology and other influencing factors are given as frequencies. Univariate regression analysis was performed to determine the influence of various parameters on TTTO.

\section{Results}

A total of 152 patients were scheduled for NACT between 2015 and 2017. After exclusion of 13 patients with insufficient records, 139 patients proved to be evaluable. Overall patient TTTO was 30.7 days $( \pm 11.8)$. TTTO for standard therapy patients was 29.4 days $( \pm 12.4)$ and 33.1 days $( \pm 10.4)$ for study patients (Fig. 1$)$. The time difference of 3.7 days was not statistically significant $(p=0.061$; Fig. 1, Table 1).

The patients' average age was 53 years $( \pm 13)$. Patients receiving standard therapy were older with a mean age of 55 years $( \pm 14)$ compared to patients receiving study therapy with 49 years $( \pm 11)$. Table 2 illustrates the distribution of tumor stage, pathology, histological subtypes, and centricity.

Most of the patients had cT1/T2 tumors $(112 / 139=$ $80.6 \%)$ and a lymph node status of cN0 (77/139= $55.4 \%)$. None of the patients had primary metastasis. The predominant part was classified as NST carcinoma $(126 / 139=90.6 \%)$. The luminal A subtype was rare $(6 / 139=4.3 \%)$, whereas luminal B, Her2-positive and triple-negative subtypes were equally distributed (32.4, 33.8 , and $29.5 \%$ ). Fourteen percent of the patients had multiple tumor lesions, whether multifocal, multicentric, or bilateral BC.

Ninety patients $(64.7 \%)$ were treated with standard therapies according to guidelines. Forty-nine (35.3\%) re-

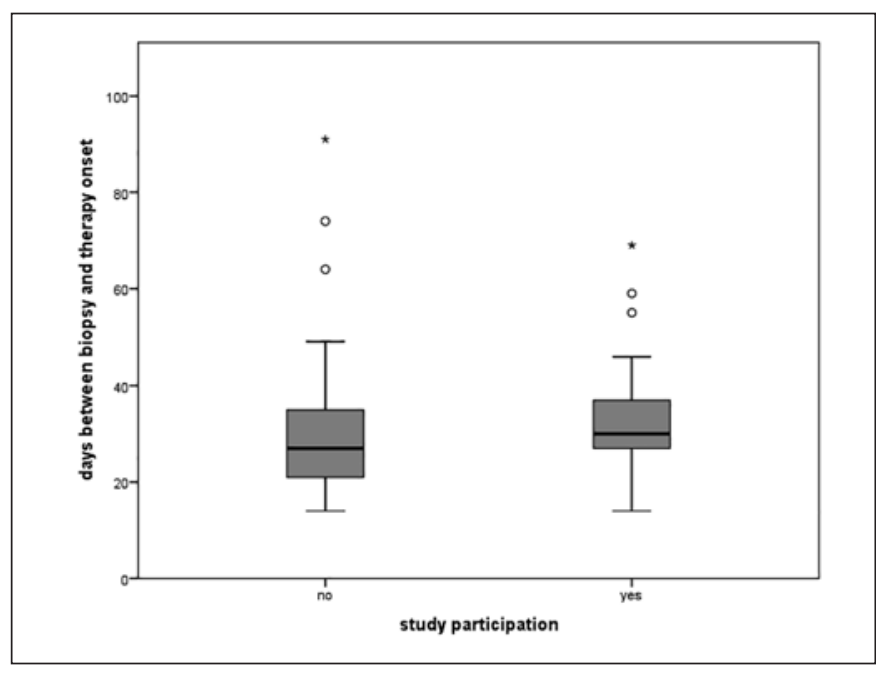

Fig. 1. Time interval between the day of core biopsy of breast tumor and the first day of systemic therapy application. Time to treatment onset was $30.7 \pm 11.8$ days. Standard therapy patients started after $29.4 \pm 12.4$ days; patients recruited to clinical studies had an interval of $33.1 \pm 10.4$ days. There was no significant time difference $(p=0.061)$.

ceived study therapies. The main part was treated in the GAIN II protocol (https://clinicaltrials.gov/ct2/show/ NCT01690702) followed by GeparOcto (https://clinicaltrials.gov/ct2/show/NCT02125344).

Prior to the onset of therapy, the patients had a mean of $5( \pm 1.9)$ clinical presentations, 4 of them $( \pm 1.8)$ on outpatient and $1( \pm 0.5)$ on inpatient basis, with no significant differences between the study- and the non-study group. Details are given in Table 3.

The reason for inpatient procedures was mostly (71\%) sentinel-node biopsy accompanied by port-implantation into the cephalic vein to facilitate systemic therapy. All in all, 69 patients (71\%) received a sentinelnode biopsy before the start of NACT. In rare cases $(11.3 \%)$, the patient had to be asked to agree to inpatient magnetic resonance examination when lobular carcinoma had been diagnosed or multicentricity was suspected. In some other rare cases, surgical procedures following a fertility preservation program, for example, endoscopic harvesting of ovarian tissue or clarification of suspicious ovarian ultrasound findings before starting NACT was required.

Possible factors delaying TTTO, such as pathology procedures, reproductive medicine advice, or procedures and genetic counseling, were gathered carefully. External pathology was supplied in 19.4\% (27/139; study 10/49= $20.4 \%$ and standard $17 / 90=18.9 \%)$. Reproduction advice was given in $10.8 \%(15 / 139$; study $8 / 49=16.3 \%$ and standard $7 / 90=7.8 \%)$. Genetic counseling happened in $26.6 \%$ $(37 / 139$; study $16 / 49=32.7 \%$ and standard $21 / 90=$ $23.3 \%)$. The particular frequencies of these factors were 
Table 1. Univariate regression analysis: factors possibly influencing TTTO (time to treatment onset) have been analyzed

\begin{tabular}{llccc}
\hline Parameter & $\begin{array}{l}\text { Significance, } \\
\text { p value }\end{array}$ & $\begin{array}{l}95 \% \text { CI } \\
\text { lower value }\end{array}$ & $\begin{array}{l}\text { 95\% CI } \\
\text { upper value }\end{array}$ & $\begin{array}{l}\text { Regression } \\
\text { coefficient }\end{array}$ \\
\hline Age (years) & 0.661 & -0.09 & 0.17 & 0.03 \\
Study participation & 0.061 & -0.08 & 7.68 & 3.76 \\
All clinical presentations & $\mathbf{0 . 0 2 1}$ & 0.48 & 3.61 & 1.98 \\
Presentation on outpatient basis & $\mathbf{0 . 0 4 7}$ & 0.27 & 3.34 & 1.71 \\
Presentation on inpatient basis & $\mathbf{0 . 0 1 7}$ & 1.01 & 7.73 & 4.38 \\
Histology ex domo & 0.190 & -1.35 & 8.91 & 3.56 \\
IVF counseling & 0.927 & -5.28 & 6.30 & 0.27 \\
IVF treatment & 0.241 & -3.18 & 15.09 & 5.03 \\
Genetic counseling & 0.400 & -5.45 & 2.30 & -1.64 \\
Second breast cancer & $\mathbf{0 . 0 0 1}$ & -14.26 & -3.89 & -8.18 \\
Multiple tumors (e.g., bilateral) & 0.805 & -4.87 & 7.40 & 0.85 \\
\hline
\end{tabular}

Clinical presentations ( $p=0.021)$ were found to have a significant influence, whether on out- $(p=0.047)$ or inpatient $(p=0.017)$ basis. A second breast cancer also had a significant influence on TTTO $(p=0.001)$.

similarly distributed in terms of study or non-study participation. The total frequencies of these factors were taken for regression analysis.

Four patients had secondary contralateral cancer as exclusion criterion for study participation, and the same held true for a patient with simultaneous urothelium carcinoma. These patients were scheduled for standard therapy.

All factors that might influence TTTO were analyzed by univariate regression analysis (Table 1 ). Study participation delayed TTTO by an average of 4 days, but this was not statistically significant $(p=0.061)$. Age had no significant influence on TTTO, and the same was valid for the site of pathology, additional reproductive medicine advice or treatment, and genetic counseling. Multiple uni- or bilateral tumors in the breast did not affect TTTO either.

However, significant influence on TTTO was seen with regard to the total number of pretherapeutic presentations $(p=0.021)$, number of outpatient presentations ( $p$ $=0.047)$, and inpatient procedures $(p=0.017)$. Figure 2 underlines that every pretherapeutic presentation lengthened TTTO.

In detail, we found outpatient visits delaying TTTO by an average of 2 days, and any inpatient activity added 4 days to TTTO. In contrast, a second contralateral BC after a history of prior BC diminished TTTO by 8 days.

\section{Discussion}

In a single-center study at the Department of Gynecology, Saarland University Medical School, we found an average 31-day interval from the date of diagnosis to the beginning of neoadjuvant therapy in BC. Possible factors influencing the TTTO were investigated.

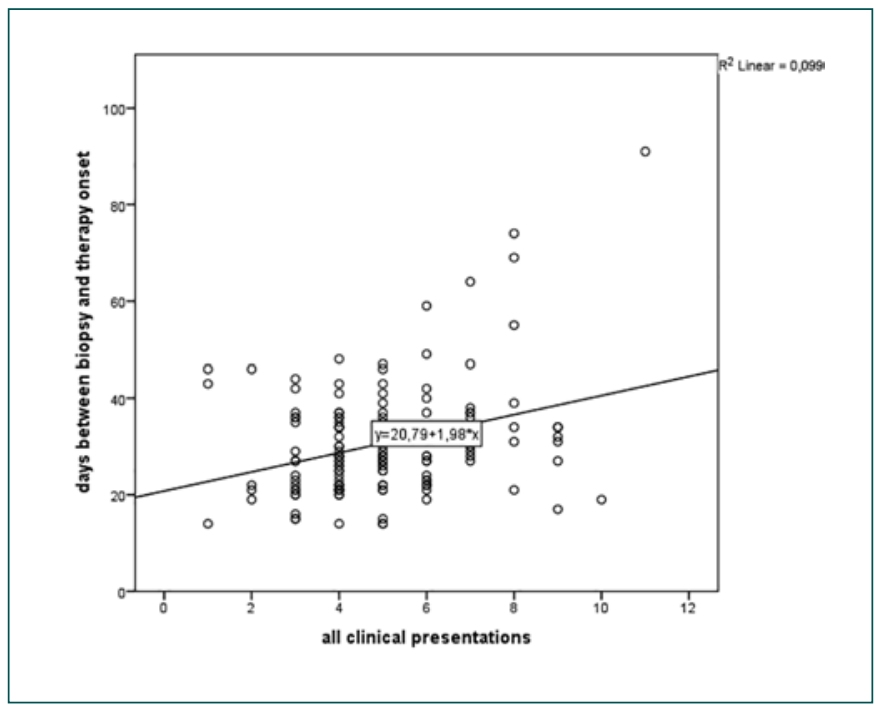

Fig. 2. Graphical representation using the results of the univariate regression analysis of all clinical presentations regarding the time to treatment onset.

One delaying factor might be age at diagnosis. Von Waldenfels et al. [5] underlined that even older patients may profit by NACT, and the desired complete remission does not depend on the patient's age, but on tumor-specific prognostic factors [6]. On the other hand, delay of systemic therapy onset might impair outcome and survival in BC patients. In our study, age had no influence on TTTO. Next to that, patients scheduled for NACT trial protocol were younger compared to patients receiving standard therapy. This might be due to advice bias, as it is known that younger patients benefit more from NACT compared to older ones [7]. In accordance with that, Hamelinck et al. [8] showed that patients $<65$ years old 
Table 2. Distribution of tumor stage, histological subtypes, and centricity

\begin{tabular}{|c|c|c|}
\hline & $\begin{array}{l}\text { Absolute } \\
\text { frequencies, } \\
n\end{array}$ & $\begin{array}{l}\text { Cumulative } \\
\text { frequencies, } \\
\%\end{array}$ \\
\hline \multicolumn{3}{|l|}{$c T$} \\
\hline 1a & 0 & 0 \\
\hline $1 b$ & 7 & 5.0 \\
\hline $1 \mathrm{c}$ & 49 & 35.3 \\
\hline 2 & 56 & 40.3 \\
\hline 3 & 10 & 7.2 \\
\hline 4 & 10 & 7.2 \\
\hline $4 \mathrm{a}$ & 0 & 0 \\
\hline $4 \mathrm{~b}$ & 2 & 1.4 \\
\hline $4 d$ & 3 & 2.2 \\
\hline $\mathrm{X}$ & 2 & 1.4 \\
\hline \multicolumn{3}{|l|}{$c N$} \\
\hline 0 & 77 & 55.4 \\
\hline 1 & 39 & 28.1 \\
\hline 2 & 20 & 14.4 \\
\hline 3 & 2 & 1.4 \\
\hline $3 c$ & 1 & 0.7 \\
\hline \multicolumn{3}{|l|}{ M } \\
\hline 0 & 139 & 100 \\
\hline \multicolumn{3}{|l|}{ Histology } \\
\hline Basaloid subtype & 1 & 0.7 \\
\hline Medullary characteristics & 2 & 1.4 \\
\hline Metaplastic & 1 & 0.7 \\
\hline Mucinous & 1 & 0.7 \\
\hline NST & 126 & 90.6 \\
\hline NST, inflammatory & 7 & 5.0 \\
\hline Plate epithelium differentiation & 1 & 0.7 \\
\hline \multicolumn{3}{|l|}{ Subtype } \\
\hline \multicolumn{3}{|l|}{ Luminal A ER+, PR+, Her2neu neg, } \\
\hline Ki67 $\leq 15 \%$ & 6 & 4.3 \\
\hline \multicolumn{3}{|l|}{ Luminal B ER+, PR+, Her2neu neg, } \\
\hline Ki67 $>15 \%$ & 45 & 32.4 \\
\hline HER2 like ER, $\pm P R \pm$, Her2neu pos & 47 & 33.8 \\
\hline Triple negative & 41 & 29.5 \\
\hline \multicolumn{3}{|c|}{ Multiple tumor masses (multifocal, multicentricity, bilateral) } \\
\hline All patients & 19 & 13.7 \\
\hline Non-study group & 12 & 13.3 \\
\hline Study group & 7 & 14.3 \\
\hline
\end{tabular}

tend to be informed better about possible therapy strategies and prospective studies, whereas older patients prefer standard procedures.

Surprisingly, we observed in our study population that study participation did not significantly prolong TTTO. Nevertheless, a mean prolongation of TTTO with 3.7 days is remarkable and may be caused by administrative and structural restraints like checking of inclusion/exclusion criteria, control of morphologic and biologic assessment, metastasis status, organ function by controlling lab data, and so on. Control mechanisms for study enroll-
Table 3. Number of clinical presentations of the patients between the day of core biopsy of breast tumor and the first day of systemic therapy application

\begin{tabular}{ll}
\hline All clinical presentations & \\
All patients & $5.01 \pm 1.88$ \\
Non-study group & $4.92 \pm 1.85$ \\
Study group & $5.16 \pm 1.93$ \\
\hline Outpatient presentations & \\
All patients & $4.28 \pm 1.82$ \\
Non-study group & $4.17 \pm 1.83$ \\
Study group & $4.49 \pm 1.80$ \\
\hline Inpatient presentations & \\
All patients & $0.73 \pm 0.52$ \\
Non-study group & $0.77 \pm 0.50$ \\
Study group & $0.67 \pm 0.56$ \\
\hline
\end{tabular}

Clinical presentations were divided into all presentations and presentations on outpatient and inpatient basis. Data are presented as mean $\pm \mathrm{SD}$.

ment aim at a low dropout rate of patients considered for study therapies. However, optimization of administration pathways of the study center will remain a permanent challenge to achieve the shortest possible TTTO.

One of the most important results of this study was that any further clinical presentation prolongs TTTO significantly. So, the number of presentations should be limited by merging appointments for imaging and lab diagnosis with dates for medical advice and therapy planning. Inpatient presentations prolonged TTTO ( 4 days) more than outpatient presentations ( $\sim 2$ days). Inpatient procedures should thus be substituted by outpatient procedures whenever possible. Breast care centers should thus be encouraged to optimize clinical pathways to accelerate therapy onset. Talking to the patient, arranging appointments, giving medical advice and signing papers is timeconsuming, and this time is increased by documentation of all measures taken. This should be considered to ensure adequate covering treatment funds. Beckmann et al. [9] examined documentation efforts in several BC centers. He reported expenses of EUR 350-1,100 exclusively for documentation from diagnosis to follow-up in BC patients, with $57 \%$ of these efforts having been done by doctors [9]. Obviously, these efforts could and should be lowered by structural changes and personal competence.

Patients that underwent mammography screening and core biopsy at our institution were expected to have a shorter TTTO, due to a presumed shortening of administrative procedures and clinical presentations. However, this was not the case. Site of pathology examination did not matter for TTTO in our study population. In Germany, a mammography screening program has been enabled for all patients aged between 50 and 69 years by federal law. Participation in the program is supposed to 
lead to mortality reduction [10]. $19.4 \%$ of our patients received core biopsy externally, $7.2 \%$ as part of mammography screening. The patients presented themselves in our breast center with finished histological results. In our population, the site of pathology examination had no significant influence on TTTO. To our knowledge, comparable literature data have not been published yet.

Younger patients need genetic counseling and possibly BRCA $1 / 2$ testing in order to get thorough information for systemic therapy decision [11]. Furthermore, patients in the reproductive age need advice regarding fertility protection and cryopreservation techniques [12]. As NACT will provide a better prognosis for young patients with HER2-like or triple-negative tumors [13], advice regarding helpful reproduction methods is important. Hormone receptor-positive patients of fertile age need fertility protection advice as well, as a worsening of their prognosis is not likely by future pregnancy [14]. In any case, future pregnancy is up to the patient, but planning should be postponed until the complete $\mathrm{BC}$ therapy has been finished and ovarian function has recovered. Contraceptive strategies should therefore be discussed with the patient before therapy onset $[15,16]$. These procedures are time consuming, presumably delaying TTTO. In this study, $37 \%$ had genetic counseling and $10.8 \%$ wanted reproduction advice. In 6 cases, fertility protection procedures were carried out before systemic therapy onset. No significant delay of TTTO could be seen by counseling or treatments for fertility preservation. A reason for this could be the proximity and close cooperation between the breast center and the fertility center. This simplifies the access for consultation for fertility preservation. Chien et al. [17] showed similar results investigating fertility protection by ovarian stimulation in young $\mathrm{BC}$ patients. $\mathrm{He}$ reported a 40-day TTTO interval without significant differences between patients with or without an ovarian stimulation program [17]. We observed a TTTO of 31 days, and in line with the results of Chien et al. [17], fertility protection did not compromise TTTO.

All efforts to reduce TTTO should be based on relevance for outcome. Loibl et al. [18] analyzed the pooled data of 9,127 patients in NACT studies and reported that TTTO below or beyond 4 weeks did not influence pathologic complete remission or disease-free or overall survival. In contrast, several studies showed the consequences of a delayed treatment onset of adjuvant chemotherapy $[19,20]$. Patients with stage III BC had worse outcomes when time to chemotherapy was longer than 60 days compared to a therapy onset less than 30 days after surgery [20]. Consistently, Morante et al. [19] reported an increased risk for disease recurrence and death by $90 \%$ in patients with triple-negative $\mathrm{BC}$ when therapy onset exceeded 30 days after surgery. However, these studies investigated adjuvant chemotherapy settings. Available data suggest that reduction of TTTO is critical when tumor stage is high and adjuvant chemotherapy is indicated, but not as important when NACT is applied. Nevertheless, these studies showed the tremendous consequences of prolonged TTTOs. Our results may thus help to optimize clinical pathways for $\mathrm{BC}$ patients and therefore enhance future therapy outcomes.

Our study also has some limitations. This study was performed in a single certified breast care center. Clinical pathways might be different in other centers. For example, Loibl et al. [18] reported a slightly shorter median TTTO for patients receiving NACT ranging from 24 to 29 days. During the study period, a biopsy of the sentinel lymph node was standard of care in Germany before NACT was applied. Current guidelines do not support this anymore, possibly leading to a further shortening of TTTO nowadays. Not all possible delaying factors were addressed in this study. For example, clip implantations in the tumor, or outpatient clinical presentations as CT scans, MRI scans or echocardiographs can influence the TTTOs as well. Some patients might also obtain a second opinion before starting therapy.

\section{Conclusion}

In a single-center study at the Department of Gynecology, Saarland University Medical School, we found an average 31-day interval from the date of diagnosis to the beginning of neoadjuvant therapy in BC. We were looking for possible reasons which delayed the onset of NACT and detected that in- and outpatient presentations will prolong the time interval by 2 and 4 days, respectively. The challenge is to fix the diagnostic program and advisory appointments as early as possible. Thereby, the number of pretreatment presentations should be reduced and the TTTO may be shortened effectively. To our knowledge, we have been the first to show that the site of pathology had no effect on TTTO. Likewise, patient's decision to participate in a NACT study protocol will not defer the start of treatment. Therefore, study enrollment may be recommended to every patient fit for neoadjuvant therapy protocols. Age, genetic counseling, and fertility preservation advice and treatment did not delay therapy onset either, as has been reported elsewhere.

\section{Statement of Ethics}

Informed consent was obtained from every individual participant included in the study. All procedures performed in the study involving human participants were in accordance with the ethical standards of the institutional and/or national research committee and with the 1964 Helsinki Declaration and its later amendments or comparable ethical standards. 


\section{Disclosure Statement}

All authors declare that they have no conflict of interest.

\section{Funding Sources}

This study was not funded.

\section{References}

1 Robert Koch-Institut. Bericht zum Krebsgeschehen in Deutschland 2016. Bericht zum Krebsgeschehen Deutschl; 2016. pp. 16-77.

2 Leitlinienprogramm Onkologie. Interdisziplinäre S3-Leitlinie für die Diagnostik. Therapie und Nachsorge des Mammakarzinoms; 2012. pp. 32-45.

3 Sardanelli F, Aase HS, Álvarez M, Azavedo E, Baarslag HJ, Balleyguier C, et al. Position paper on screening for breast cancer by the European Society of Breast Imaging (EUSOBI) and 30 national breast radiology bodies from Austria, Belgium, Bosnia and Herzegovina, Bulgaria, Croatia, Czech Republic, Denmark, Estonia, Finland, France, Germany, Greece, Hungary, Iceland, Ireland, Italy, Israel, Lithuania, Moldova, The Netherlands, Norway, Poland, Portugal, Romania, Serbia, Slovakia, Spain, Sweden, Switzerland and Turkey. Eur Radiol. 2017 Jul;27(7):2737-43.

4 Kaufmann M, von Minckwitz G, Mamounas EP, Cameron D, Carey LA, Cristofanilli M, et al. Recommendations from an international consensus conference on the current status and future of neoadjuvant systemic therapy in primary breast cancer. Ann Surg Oncol. 2012 May;19(5):1508-16.

5 von Waldenfels G, Loibl S, Furlanetto J, Machleidt A, Lederer B, Denkert C, et al. Outcome after neoadjuvant chemotherapy in elderly breast cancer patients - a pooled analysis of individual patient data from eight prospectively randomized controlled trials. Oncotarget. 2018 Feb;9(20):15168-79.

6 Swain SM, Nunes R, Yoshizawa C, Rothney M, Sing AP. Quantitative Gene Expression by Recurrence Score in ER-Positive Breast Cancer, by Age. Adv Ther. 2015 Dec;32(12):122236.

7 Teshome M, Hunt KK. Neoadjuvant therapy in the treatment of breast cancer. Surg Oncol Clin N Am. 2014 Jul;23(3):505-23.
8 Hamelinck VC, Bastiaannet E, Pieterse AH, van de Velde CJ, Liefers GJ, Stiggelbout AM. Preferred and Perceived Participation of Younger and Older Patients in Decision Making About Treatment for Early Breast Cancer: A Prospective Study. Clin Breast Cancer. 2018 Apr;18(2):e245-53.

9 Beckmann M, Sell C, Aydogdu M, Brucker S, Fehm T, Janni W, et al.: Dokumentationsaufwand und verbundene Ressourcen bei Patientinnen mit einem primären Mammakarzinom - von der Primärdiagnose bis zur Abschluss der Nachsorge - Ergebnisse der multizentrischen Erhebung. Das Gesundheitswes. 2016;78:e52-e52.

10 Cedolini C, Bertozzi S, Londero AP, Bernardi $S$, Seriau L, Concina S, et al. Type of breast cancer diagnosis, screening, and survival. Clin Breast Cancer. 2014 Aug;14(4):235-40.

11 AGO Breast Group German Breast Group. Diagnostik und Therapie primärer und metastasierter Mammakarzinome Prognostische und prädiktive Faktoren Prognostische und prädiktive Faktoren. Leitlinienprogr Onkol www.ago-online.de/fileadmin/downloads/leitlinien/mamma/2018-03/Gesamt_ deutsch/Alle_aktuellen_Empfehlungen_2018.pdf (December 14, 2018).

12 Menen RS, Hunt KK. Considerations for the Treatment of Young Patients with Breast Cancer. Breast J. 2016 Nov;22(6):667-72.

13 Loibl S, Jackisch C, Lederer B, Untch M, Paepke S, Kümmel S, et al. Outcome after neoadjuvant chemotherapy in young breast cancer patients: a pooled analysis of individual patient data from eight prospectively randomized controlled trials. Breast Cancer Res Treat. 2015 Jul;152(2):377-87.
14 Azim HA Jr, Kroman N, Paesmans M, Gelber S, Rotmensz N, Ameye L, et al. Prognostic impact of pregnancy after breast cancer according to estrogen receptor status: a multicenter retrospective study. J Clin Oncol. 2013 Jan; 31(1):73-9.

15 Edinburgh Cancer Centre Western General Hospital. Contraception: Advice for People Receiving Chemotherapy. Leafl Edinburgh http//www.scan.scot.nhs.uk/Search/Results. aspx?k=contraception (December 14, 2018).

16 Beksac K, Orgul G, Ozyuncu O, Yurdakok M, Altundag K, Beksac MS. Chemotherapy during pregnancy: cases of Hodgkin's and nonHodgkin's lymphoma, chronic myeloid leukemia, breast cancer, nasopharyngeal cancer, and choriocarcinoma. Oncol Res Treat. 2017; 40(7-8):441-5.

17 Chien AJ, Chambers J, Mcauley F, Kaplan T, Letourneau J, Hwang J, et al. Fertility preservation with ovarian stimulation and time to treatment in women with stage II-III breast cancer receiving neoadjuvant therapy. Breast Cancer Res Treat. 2017 Aug;165(1):151-9.

18 Loibl S, Werutsky G, Nekljudova V, Seiler S, Blohmer JU, Denkert C, et al. Impact in delay of start of chemotherapy and surgery on pCR and survival in breast cancer: A pooled analysis of individual patient data from six prospectively randomized neoadjuvant trials. J Clin Oncol. 2017;35 15_suppl:571.

19 Morante Z, Ruiz R. De la Cruz - Ku G, Namuche F, Mantilla R, Lujan M, et al.: Impact of the delayed initiation of adjuvant chemotherapy in the outcomes of triple negative breast cancer. Available from: https://www. ascopost.com/News/59545.

20 Gagliato DM, Gonzalez-Angulo AM, Lei X, Theriault RL, Giordano SH, Valero V, et al. Clinical impact of delaying initiation of adjuvant chemotherapy in patients with breast cancer. J Clin Oncol. 2014 Mar;32(8):735-44. 